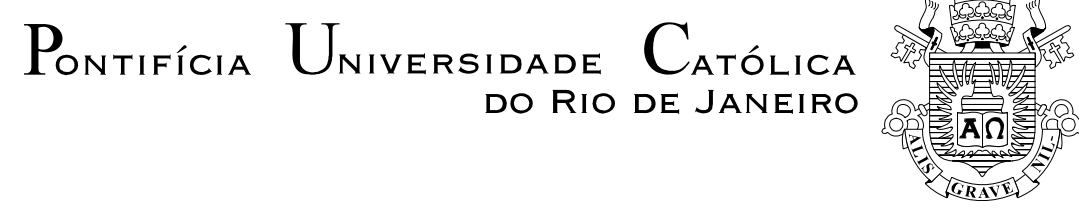

Tiago Mendes Dantas

Modelo Tempo-Frequência para Previsão de Curto Prazo de Velocidade de Vento

Dissertação de Mestrado

Dissertação apresentada ao Programa de Pós-Graduação em Engenharia Elétrica da PUC-Rio como requisito parcial para obtenção do título de Mestre em Engenharia Elétrica.

Orientador: Prof. Reinaldo Castro Souza

Rio de Janeiro

Agosto de 2011 


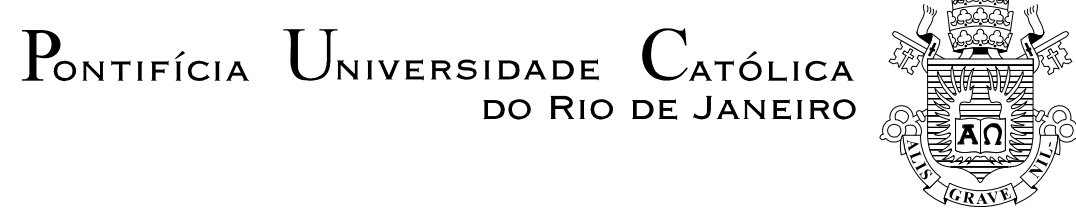

Tiago Mendes Dantas

\section{Modelo Tempo-Frequência para Previsão de Curto Prazo de Velocidade de Vento}

Dissertação de Mestrado apresentada como requisito parcial para obtenção do grau de Mestre pelo Programa de Pós-Graduação em Engenharia Elétrica do Departamento de Engenharia Elétrica do Centro Técnico Científico da PUC-Rio. Aprovada pela Comissão Examinadora abaixo assinada.

Prof. Reinaldo Castro Souza

Orientador

Departamento de Engenharia Elétrica - PUC-Rio

Prof. José Francisco Moreira Pessanha

UERJ

Prof. André Luís Marques Marcato

UFJF

Prof. José Eugenio Leal Coordenador Setorial do Centro

Técnico Científico

Rio de Janeiro, 31 de agosto de 2011 
Todos os direitos reservados. É proibida a reprodução total ou parcial do trabalho sem autorização da universidade, do autor e do orientador.

\section{Tiago Mendes Dantas}

Graduou-se como Bacharel em Estatísticas na Escola Nacional de Ciências Estatísticas em 2009.

Ficha Catalográfica

Dantas, Tiago

Modelo Tempo-Frequência para Previsão de Curto Prazo de Velocidade de Vento / Tiago Mendes Dantas; orientador: Reinaldo Castro Souza. - Rio de Janeiro : PUC-Rio, Departamento de Engenharia Elétrica, 2011.

v., 69 f: il. ; $29,7 \mathrm{~cm}$

1. Dissertação (mestrado) - Pontifícia Universidade Católica do Rio de Janeiro, Departamento de Engenharia Elétrica.

Inclui referências bibliográficas.

1. Engenharia Elétrica - Tese. 2. Energia Eólica. 3. Velocidade de Vento. 4. Previsão. 5. Memória Longa. I. Souza, Reinaldo Castro Souza. II. Pontifícia Universidade Católica do Rio de Janeiro. Departamento de Engenharia Elétrica. III. Título. 


\section{Agradecimentos}

Ao meu orientador e amigo, Professor Reinaldo Castro Souza, pelas oportunidades e pela confiança depositada.

Aos professores da PUC-Rio por todos os ensinamentos durante esses dois anos.

À minha família e sobretudo aos meus pais, Sidnei e Célia, pelo apoio e carinho durante todos os momentos.

Aos amigos especiais, Lincoln e Heloisa, que tiveram contribuição direta na elaboração dessa dissertação e na minha vida.

A todos os amigos que torceram por mim de perto ou de longe. A Deus. 


\section{Resumo}

Dantas, Tiago Mendes; Souza, Reinaldo Castro (Orientador). Modelo Tempo- Frequência para Previsão de Curto Prazo de Velocidade de Vento. Rio de Janeiro, 2011. 69p. Dissertação de Mestrado Departamento de Engenharia Elétrica, Pontifícia Universidade Católica do Rio de Janeiro.

A quantidade de energia gerada através de energia eólica está aumentando no mundo todo. O Brasil tem um enorme potencial devido a sua localização geográfica e governo brasileiro d’a claros sinais de que está propenso a investir neste tipo de energia. Previsões precisas de velocidade vento são essenciais para a operação planejamento do sistema elétrico de energia. Este trabalho tem como objetivo fazer previsões mais precisas na Nordeste do Brasil. Para isso, usamos um modelo que leva em conta as características diárias e o comportamento da memória longa. O modelo aplicado nesta região particular mostrou-se mais preciso que o modelo de persistência e outros modelos (por exemplo, modelo híbrido neuro-fuzzy)

\section{Palavras-chave}

Energia Eólica; Velocidade de Vento; Previsão; Memória Longa. 


\section{Abstract}

Dantas, Tiago Mendes; Souza, Reinaldo Castro (Advisor). TimeFrequency model for short term wind speed forecasting. Rio de Janeiro, 2011. 69p. MSc Dissertation - Departamento de Engenharia Elétrica, Pontifícia Universidade Católica do Rio de Janeiro.

The amount of energy generated by wind sources is increasing all over the world. Brazil has a huge potential due to its geographic localization and the Brazilian Government has given a clear signal that is prone to invest in this kind of energy. Accurate wind speed forecasts are essential in the operation planning for the electrical wind power system. This work aims to make more accurate forecasts in the northeast of Brazil. To do so, we use a model that takes into account the daily characteristics and the long memory behavior. The model applied in this particular region proved to be more accurate than the persistence model and other models.

\section{Keywords}

Wind energy; Wind speed; Forecasting; Long memory. 


\section{Sumário}

1 Introdução 11

1.1 Métodos de Previsão de Vento 14

2 Base de Dados $\quad 16$

3 Metodologia $\quad 18$

3.1 Processos Estocásticos 21

3.2 Previsão de Séries Temporais 22

3.3 Método Ingênuo 23

3.4 Metodologia Box e Jenkins 24

3.5 Modelo ARFIMA 28

3.6 Análise Harmônica 33

3.7 Métricas de Comparação 38

4 Análise Exploratória $\quad 39$

5 Modelagem $\quad 47$

5.1 Aplicação do modelo 50

5.2 Comparação dos resultados $\quad 60$

6 Considerações Finais $\quad 63$

$\begin{array}{lll}6.1 & \text { Sugestões } & 64\end{array}$

7 Referências Bibliográficas $\quad 66$ 


\section{Lista de figuras}

1.1 Aerogeradores 11

1.2 Distribuição de energia eólica mundial em $2010 \quad 12$

1.3 Potencial eólico no Nordeste 13

3.1 Entrada de ruído branco e geração de série temporal via filtro linear (geração de série temporal) 24

3.2 Entrada da série temporal e saída de ruído branco via filtro linear (análise de série temporal) 25

4.1 Série de dados em São João do Cariri - PB 40

4.2 Velocidade média horária do vento em São João do Cariri - PB 41

4.3 Periodograma dos dados em São João do Cariri - PB 41

4.4 Velocidade média mensal do vendo em São João do Cariri - PB 42

4.5 Função de autocorrelação da série de São João do Cariri - PB 43

4.6 Função de autocorrelação parcial da série de São João do Cariri - PB 43

4.7 Histograma da série de São João do Cariri - PB 44

4.8 Boxplot da série de São João do Cariri - PB 45

4.9 QQ-Plot da série de São João do Cariri - PB 45

5.1 Resíduos 52

5.2 Função de autocorrelação dos resíduos 53

5.3 Função de autocorrelação parcial dos resíduos 53

5.4 Histograma dos resíduos 54

5.5 Q-Q Plot dos resíduos 54

5.6 Previsão 1 passo à frente e valores observados 55

5.7 Previsão 2 passos à frente e valores observados 56

5.8 Previsão 3 passos à frente e valores observados 56

5.9 Previsão 4 passos à frente e valores observados 57

5.10 Previsão 5 passos à frente e valores observados 57

5.11 Previsão 6 passos à frente e valores observados 57

5.12 Evolução do MAPE na primeira semana de 2007

5.13 Previsão 24 passos à frente e valores observados 59

5.14 Previsões 1, 6 e 24 passo à frente e valores observados 60

5.15 Comparação: Modelo Proposto e Modelo harmônico 60

5.16 Comparação: Modelo Proposto e Modelo ARFIMA 61

5.17 Comparação: Modelo Proposto e Modelo Persistence 61 


\section{Lista de tabelas}

2.1 Estações do projeto SONDA 16

4.1 Medidas de resumo da série temporal de São João do Cariri - PB 39

4.2 Teste de estacionariedade Augmented Dickey - Fuller 40

4.3 Teste de normalidade 46

5.1 Coeficientes estimados pelo modelo 51

5.2 Resumo dos Resíduos $\quad 52$

5.3 Métricas de comparação 56

5.4 Métricas de comparação 58

5.5 Métricas de comparação $\quad 59$

5.6 Métricas de comparação entre os modelos 61 
"The answer, my friend, is blowin' in the wind The answer is blowin' in the wind"

Bob Dylan, 